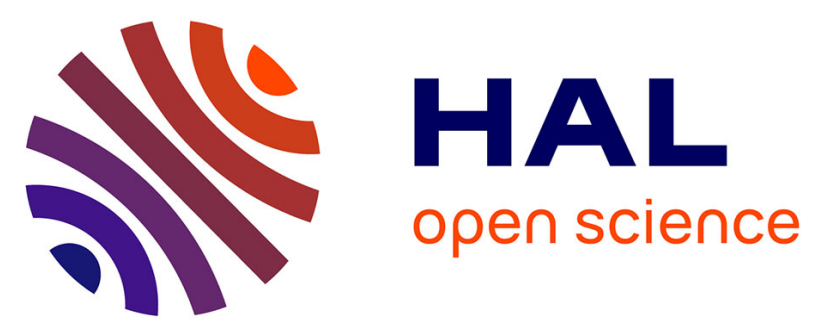

\title{
Long-term outcomes in patients with PET-predicted poor-responsive HER2-positive breast cancer treated with neoadjuvant bevacizumab added to trastuzumab and docetaxel: 5-year follow-up of the randomised Avataxher study
}

Benoit Coudert, Jean-Yves Pierga, Marie-Ange Mouret-Reynier, Kaldoun

Kerrou, Jean-Marc Ferrero, Thierry Petit, Fanny Le Du, Pierre-François

Dupré, Thomas Bachelot, Philippe Gabelle, et al.

\section{To cite this version:}

Benoit Coudert, Jean-Yves Pierga, Marie-Ange Mouret-Reynier, Kaldoun Kerrou, Jean-Marc Ferrero, et al.. Long-term outcomes in patients with PET-predicted poor-responsive HER2-positive breast cancer treated with neoadjuvant bevacizumab added to trastuzumab and docetaxel: 5-year follow-up of the randomised Avataxher study. EClinicalMedicine, 2020, 28, pp.100566. 10.1016/j.eclinm.2020.100566 . hal-03244169

\section{HAL Id: hal-03244169 \\ https://hal.sorbonne-universite.fr/hal-03244169}

Submitted on 1 Jun 2021

HAL is a multi-disciplinary open access archive for the deposit and dissemination of scientific research documents, whether they are published or not. The documents may come from teaching and research institutions in France or abroad, or from public or private research centers.
L'archive ouverte pluridisciplinaire HAL, est destinée au dépôt et à la diffusion de documents scientifiques de niveau recherche, publiés ou non, émanant des établissements d'enseignement et de recherche français ou étrangers, des laboratoires publics ou privés. 
Research Paper

\title{
Long-term outcomes in patients with PET-predicted poor-responsive HER2-positive breast cancer treated with neoadjuvant bevacizumab added to trastuzumab and docetaxel: 5-year follow-up of the randomised Avataxher study
}

\author{
Bruno Coudert ${ }^{\mathrm{a}, *}$, Jean-Yves Pierga ${ }^{\mathrm{b}}$, Marie-Ange Mouret-Reynier ${ }^{\mathrm{c}}$, Kaldoun Kerrou ${ }^{\mathrm{d}}$, \\ Jean-Marc Ferrero ${ }^{\mathrm{e}}$, Thierry Petit ${ }^{\mathrm{f}}$, Fanny Le Du ${ }^{\mathrm{g}}$, Pierre-François Dupré ${ }^{\mathrm{h}}$, Thomas Bachelot ${ }^{\mathrm{i}}$, \\ Philippe Gabelle ${ }^{\mathrm{j}}$, Marie-Pierre Chauvet ${ }^{\mathrm{k}}$, David Coeffic ${ }^{\mathrm{l}}$, Catherine Barbe ${ }^{\mathrm{m}}$, \\ Jean-Briac Prevost ${ }^{\mathrm{n}}$, Gilles Paintaud ${ }^{\mathrm{O}}$, Gilles Thibault ${ }^{\mathrm{o}}$, Abdennour Ferhat $^{\mathrm{p}}$, Julien Dupin ${ }^{\mathrm{p}}$, \\ Alina Berriolo-Riedinger ${ }^{a}$, Laurent Arnould ${ }^{a}$
}

\footnotetext{
${ }^{a}$ Centre Georges-Francois Leclerc, Dijon, France

${ }^{\mathrm{b}}$ Institut Curie, Université de Paris, Paris, France

${ }^{\mathrm{c}}$ Centre Jean Perrin, Clermont Ferrand, France

${ }^{\mathrm{d}}$ Hopital Tenon, Paris, France

e Centre Antoine Lacassagne, Nice, France

${ }^{\mathrm{f}}$ Centre Paul Strauss, Strasbourg, France

${ }^{g}$ Centre Eugène Marquis, Rennes, France

${ }^{\text {h }}$ Centre Hospitalier Universitaire Augustin-Morvan, Brest, France

${ }^{i}$ Centre Léon Berard, Lyon, France

Institut Daniel Hollard, Grenoble, France

${ }^{\mathrm{k}}$ Centre Oscar Lambret, Lille, France

${ }^{1}$ Polyclinique Courlancy, Institut du Cancer Courlancy Reims, France

${ }^{\mathrm{m}}$ CHU Bretonneau, Tours, France

${ }^{n}$ Centre Pierre Curie, Beuvry, France

${ }^{\circ}$ Université de Tours, CHRU de Tours, Tours, France

${ }^{\mathrm{p}}$ Roche France S.A.S., Boulogne Billancourt, France
}

\section{A R T I C L E I N F O}

\section{Article History:}

Received 31 March 2020

Revised 10 September 2020

Accepted 11 September 2020

Available online $\mathrm{xxx}$

\section{Keywords:}

Her-2 positive breast cancer

Bevacizumab

Neoadjuvant

Positron emission tomography

Early pet assessment

$\Delta$ suvmax

pathological complete response

Long-term follow-up

\section{A B S T R A C T}

Background: The open-label, randomised Phase 2 AVATAXHER study (NCT01142778) demonstrated that early PET assessment identified HER2-positive breast cancer patients who responded poorly to neoadjuvant docetaxel plus trastuzumab. Adding neoadjuvant bevacizumab for PET-predicted poor-responders improved pathological complete response (pCR) rates (43.8\% vs $24.0 \%$ ). We investigated long-term study outcomes. Methods: Patients were treated in three groups. All patients initially received two cycles of standard neoadjuvant therapy with $\left[{ }^{18} \mathrm{~F}\right]-$ FDG PET conducted before each cycle. Those with $\geq 70 \%$ change in the maximum standardised uptake value ( $\triangle$ SUVmax) received four further cycles of standard neoadjuvant therapy (PET responders). PET-predicted poor-responders ( $\triangle$ SUVmax $<70 \%$ ) were randomised $(2: 1)$ to neoadjuvant therapy with (Group A) or without (Group B) bevacizumab for cycles 3-6. All patients received one further cycle of trastuzumab before surgery plus adjuvant trastuzumab (11 cycles).

Findings: 142 patients were randomized and treated (PET responders, $n=69$; Group A, $n=48$; Group B, $n=25)$. 5-year disease-free survival rates were $90.5 \%$ (95\% CI: 80.0-95.6\%) in PET responders, $90.2 \%(95 \% \mathrm{CI}$ : 75.9-96.2\%) in Group A, and 76.0\% (95\% CI: 54.2-88.4\%) in Group B. However, no difference was observed between randomised arms in a sensitivity analysis. During adjuvant therapy, the incidence of Grade $\geq 3$ (Group A: 25.6\%; Group B 12.5\%) and serious adverse events (Group A: 18.6\%; Group B 12.5\%) was higher in Group A vs Group B, but with no apparent effect on cardiac events.

Interpretation: In patients with HER2-positive breast cancer, an intervention based on early PET assessment and improvement of pCR does not modify disease-free survival.

\footnotetext{
* Corresponding author.

E-mail address: bcoudert@cgfl.fr (B. Coudert).
} 


\section{Introduction}

Trastuzumab with/without pertuzumab anti-HER2 therapy plus taxane-containing chemotherapy is a standard of care in the neoadjuvant treatment of women with early stage HER2-positive breast cancer $[1,2]$ with the aim of improving pathological complete response (pCR) rates and facilitating surgery. Several studies have shown positron emission tomography (PET) to be an early predictor of $\mathrm{pCR}$ under neoadjuvant therapy in early breast cancer, [3-5] with recent evidence that it may also have a role in predicting long-term outcomes in these patients [6,7].

The AVATAXHER study was an open-label, randomised, phase II study that investigated the prognostic value of early PET assessment in patients with early-stage HER2-positive breast cancer [8]. The

\section{Research in context}

\section{Evidence before this study}

Prior to initiating the AVATAXHER study, PubMed, Web of Knowledge, and ClinicalTrials.gov were searched using the terms 'neoadjuvant', 'HER-positive', and 'breast cancer' to identify studies that investigated the use of ${ }^{18} \mathrm{~F}$-PET to predict response to neoadjuvant treatment. No relevant publications or studies were identified in the original search, limited to articles published between May 1, 2007 and May 1, 2012. Consequently, the AVATAXHER study was initiated. The primary analysis of AVATAXHER demonstrated that early PET assessment can identify patients with early-stage HER2-positive breast cancer who will respond poorly to standard docetaxel plus trastuzumab neoadjuvant therapy, and that adding bevacizumab to standard neoadjuvant therapy in PET-predicted poor responders improved the pathological complete response (pCR) rate at surgery (from $24.0 \%$ [95\% CI: $9.4-45.1 \%$ ] to $43.8 \%$ [95\% CI: 29.5-58.8\%]).

\section{Added value of this study}

This pre-planned 5-year follow-up analysis showed that the improved pCR rate in PET-predicted poor responders observed in the AVATAXHER study did not translate into improved longterm outcomes. The 5-year disease-free survival rates were 90.5\% (95\% CI: 80.0-95.6\%) in the PET-predicted responders ( $\triangle$ SUVmax $\geq 70 \%$ ), 90.2\% (95\% CI: 75.9-96.2\%) in PET-predicted poor responders who received additional neoadjuvant bevacizumab, and $76.0 \%$ (95\% CI: 54.2-88.4\%) in PET-predicted poor responders who received standard neoadjuvant therapy. However, a sensitivity analysis discarded any significant difference between the two randomised arms.

\section{Implications of all the available evidence}

While the results of this exploratory study require confirmation in a phase III trial, the AVATAXHER study showed the value of early PET to identify patients with HER2 positive early breast cancer that are unlikely to respond to standard neoadjuvant therapy; however, adding bevacizumab to docetaxel plus trastuzumab neoadjuvant therapy in these patients does not improve long-term outcomes. study also investigated the addition of bevacizumab, which has shown synergistic effects with trastuzumab in animal models, [9] to docetaxel plus trastuzumab neoadjuvant treatment. Clinical trials in HER2 positive breast cancer also conclude that the association of bevacizumab and trastuzumab may be synergistic and promising, but these trials are small and their conclusions have to be confirmed in larger trials [10-12]. The results of the AVATAXHER study demonstrated two key findings: first, that early PET assessment identified patients predicted to respond poorly to docetaxel plus trastuzumab neoadjuvant treatment - in patient receiving docetaxel plus trastuzumab only, the negative predictive value of $\triangle \mathrm{SUVmax}$ for predicting pCR was 75.0\%. Second, the addition of bevacizumab to neoadjuvant therapy in PET-predicted poor-responders improved pCR rates (from $24.0 \%$ to $43.8 \%$ ) [8]. These results suggest a new role for PET in the early identification of non-responders to neoadjuvant docetaxel plus trastuzumab therapy and the activity of bevacizumab in this setting.

While pCR is recognised by both the FDA and EMA as a surrogate for favourable outcome in breast cancer clinical trials, improvements in $\mathrm{PCR}$ rates have not consistently translated into long-term outcome benefit in breast cancer trials, [13-16] possibly due to differences in study design, breast cancer subtype, patient characteristics and treatment administration / sequencing [14]. Improvements in $\mathrm{pCR}$ observed after the addition of bevacizumab to neoadjuvant chemotherapy in patients with HER2-negative breast cancer [17-19] have largely not been associated with improved long-term outcomes [18,20-22]. However, one recent study in patients with HER2-positive locally advanced breast cancer treated with neoadjuvant trastuzumab plus bevacizumab found a pCR of $46 \%$ and 5-year recurrencefree survival and overall survival of $79.9 \%$ and $90.8 \%$, respectively [23]. Breast cancer subtype, dose modification according to response, and treatment sequencing may therefore all influence outcome.

Here we report the results of a pre-defined 5-year outcome analysis of the AVATAXHER study, which investigated whether the improved PCR rate observed with the addition of bevacizumab to neoadjuvant docetaxel plus trastuzumab in PET-predicted poor-responders would translate into improved long-term outcome in HER2positive breast cancer patients. The study also provided further insight into the tolerance of bevacizumab added to neoadjuvant docetaxel plus trastuzumab followed by adjuvant trastuzumab.

\section{Patients and methods}

\subsection{Study design and participants}

Full details of the design of the AVATAXHER study and inclusion criteria have been reported previously [8]. The full study protocol is available in the appendix. The study was an exploratory open-label, noncomparative, randomised phase II trial conducted in four stages (Fig. 1 and Fig. 2). The primary endpoint, reported previously, [8] was the centrally assessed pathological complete response rate, defined as complete disappearance of tumour in the breast and the axilla according to the Chevallier classification. In brief, the study population comprised women with early stage HER2-positive, histologically confirmed breast cancer (T2/T3 and NX/NO/N1) with ECOG PS 0-2, who were scheduled to receive neoadjuvant therapy and were amenable to receiving $\left[{ }^{18} \mathrm{~F}\right]-$ fluorodeoxyglucose (FDG) PET imaging prior to cycles 1 and 2.

Patients were recruited between May 19, 2010 and October 1, 2012 from 26 oncology centres in France. All enroled patients provided written informed consent before screening procedures that were specific for this study. The study was approved by the central Ethics Committee 


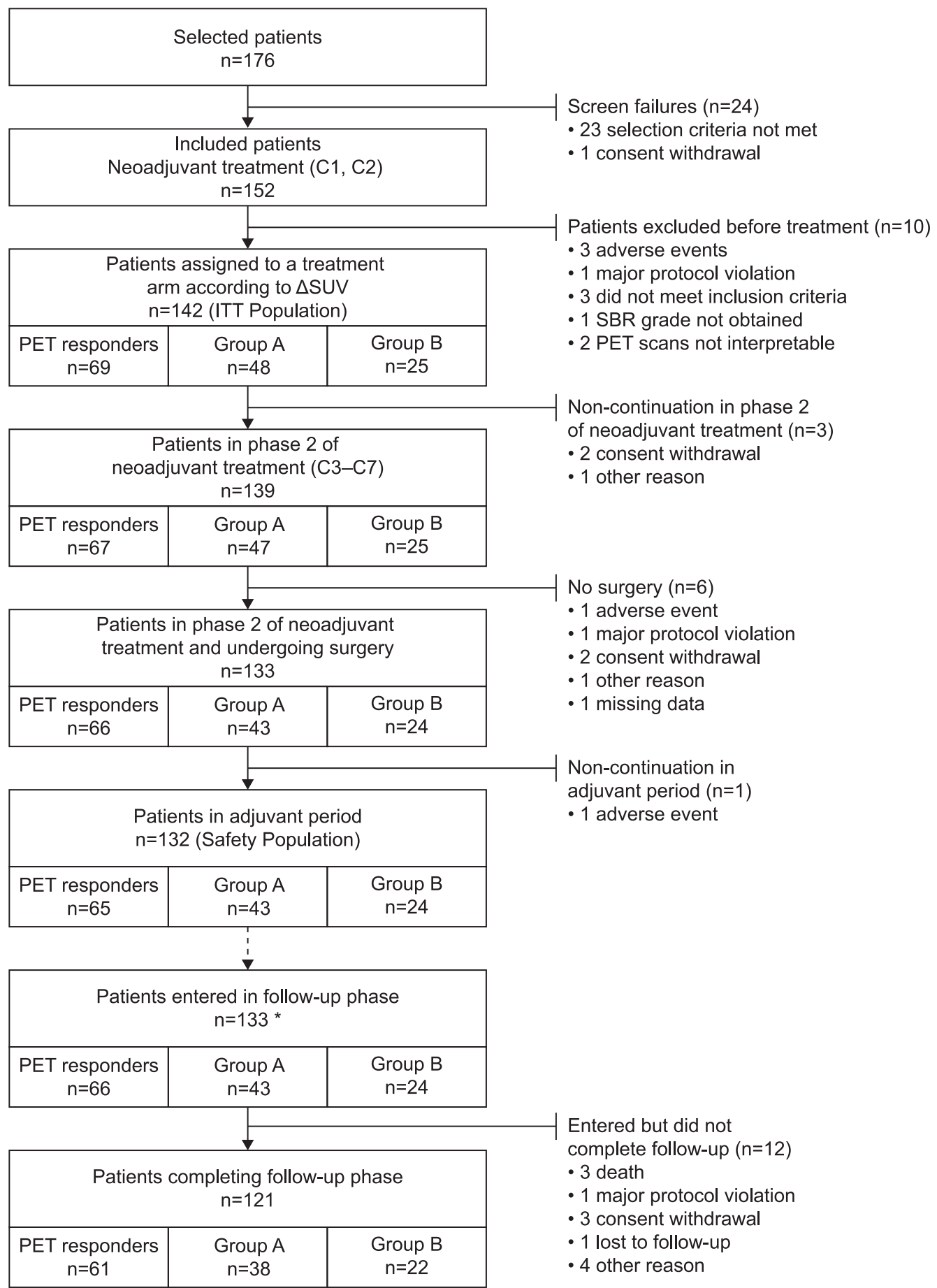

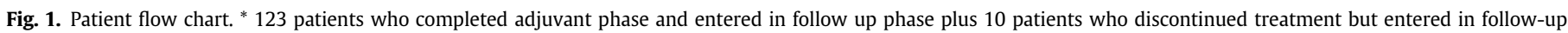
phase.

(Comité de Protection des Personnes) for all participating centres and conducted in accordance with the Declaration of Helsinki and Good Clinical Practice guidelines. The study is registered with ClinicalTrials. gov (NCT01142778) and EudraCT (2009-013410-26).

\subsection{Treatment}

In Stage 1, all patients initially received two cycles of neoadjuvant docetaxel $\left(100 \mathrm{mg} / \mathrm{m}^{2}\right)$ plus trastuzumab $(8 \mathrm{mg} / \mathrm{kg}$ in cycle 1 , and $6 \mathrm{mg} /$ $\mathrm{kg}$ thereafter), both administered intravenously (i.v.) every 3 weeks (q3w). No surgical axilla staging was allowed before neoadjuvant therapy. $\left[{ }^{18} \mathrm{~F}\right]-$ FDG PET was performed before and after the first cycle of neoadjuvant therapy (within -7 days of cycle 1 and -3 days of cycle 2) and the change in maximum standardised uptake value ( $\Delta$ SUVmax) was calculated. In Stage 2, patients with a $\Delta \mathrm{SUVmax} \geq 70 \%$ continued to receive standard docetaxel and trastuzumab therapy for cycles 3-6 (PET responders group), whereas PET-predicted poor-responders ( $\triangle$ SUVmax $<70 \%$ ) were randomised $(2: 1)$ to receive either four cycles of docetaxel and trastuzumab with (Group A) or without (Group B) bevacizumab (15 mg/kg i.v. q3w). One cycle of trastuzumab alone (cycle 7) was administered to all patients before surgery, which was performed after cycle 7 and $\geq 4$ weeks after the last infusion of cycle 6 . Following surgery, in Stage 3, patients received 11 cycles of adjuvant trastuzumab q3w with or without radiotherapy (4-6 weeks according to the site's 

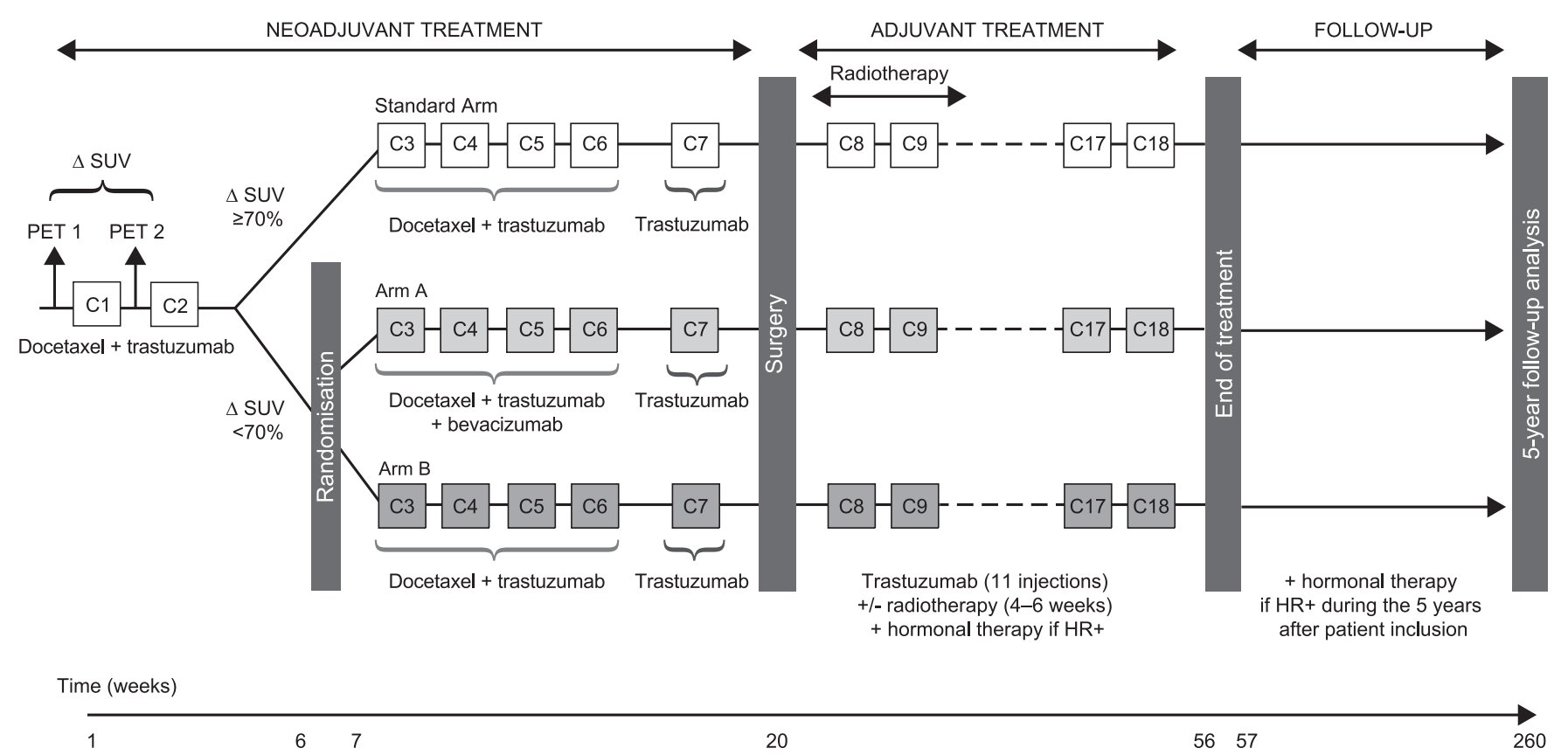

Fig. 2. AVATAXER study design.

standard practice) plus hormonal therapy in hormone receptor positive patients. Stage 4 consisted of a 5-year post-treatment follow-up during which hormonal therapy was permitted in hormone receptor positive patients only.

\subsection{Randomisation and masking}

Randomisation methods have been described in full previously [8]. Investigators and patients were aware of group assignment.

\subsection{5-year follow-up study endpoints and statistical analyses}

\subsubsection{Follow-up}

Patients were assessed during follow-up at biannual clinic visits with assessment of left ventricular ejection fraction (LVEF), chest Xray, and abdominal ultrasound, as well as yearly mammography.

\subsubsection{Efficacy outcomes}

Long-term efficacy outcomes were secondary endpoints of the AVATAXHER study and comprised disease-free survival (DFS), distant disease-free survival (DDFS), local relapse-free interval (LRFI), and OS. DFS was defined as time from first administration of neoadjuvant treatment to local recurrence, local recurrence in the ipsilateral breast following lumpectomy, regional recurrence, occurrence of distant metastases, controlateral breast cancer, second primary cancer (other than squamous or basal cell carcinoma of the skin, melanoma in situ, carcinoma in situ of the cervix, colon carcinoma in situ, or lobular carcinoma in situ of the breast), or death from any cause. LRFI was defined as the time from first administration of neoadjuvant treatment to local recurrence in the ipsilateral or controlateral breast following lumpectomy. DDFS was defined as time to distant recurrence following first administration of neoadjuvant treatment. OS was defined as time from first administration of neoadjuvant treatment to death from any cause.

\subsubsection{Safety outcomes}

Safety during the adjuvant phase was analysed in the subset of patients from the safety population $(\mathrm{N}=132)$ who received at least one dose of trastuzumab during the adjuvant phase. For safety analyses, patients were grouped according to study group. Safety outcomes included incidence of adverse events (AEs) up to 28 days after the last treatment administration. AEs were coded according to the MedDRA guidelines and their intensity graded by the National Cancer Institute Common Terminology Criteria for Adverse Events version $4 \bullet 0$. Cardiac safety was assessed according to the New York Heart Association classification.

\subsubsection{Statistics}

Determination of the sample size for the AVATAXHER study has been reported previously [8]. Efficacy analyses were conducted using the intention-to-treat (ITT) population (i.e. all patients assigned to treatment arm according to $\triangle \mathrm{SUVmax}$ and randomised). Time-toevent measures were analysed by the Kaplan-Meier method. Median values for survival parameter estimates at 5 years were calculated with 95\% CI. Patients lost to follow up were censored at the date of last assessment of the event. For patients alive at study end (premature discontinuation or final visit) censoring for OS was the last known date alive. Additional censoring was applied for LRFI (at first date of regional occurrence, occurrence of distant metastases, second primary cancer, or death without evidence of recurrence) and DDFS (at first date of local or regional recurrence, second primary cancer, contralateral cancer, or death from any cause).

A post-hoc sensitivity analysis for DFS was conducted using alternative censoring methods. In this analysis, patients who stopped treatment early (for example, due to consent withdrawal) were considered treatment failures.

All data analyses were performed using SAS version $9 \bullet 4$. This manuscript adheres to CONSORT reporting guidelines.

\subsection{Role of the funding source}

The funder of the study (Roche France) supplied trastuzumab and bevacizumab and had a role in the design and conduct of the study; collection, management, and analysis of data (including statistical analyses); interpretation of findings;. The corresponding author had full access to all the data and the final responsibility to submit for publication. All authors were involved in the critical review of the manuscript during its development and approved the final version for submission. 
Table 1

Reasons for premature study discontinuation of the study $(N=21)$.

\begin{tabular}{lllll}
\hline & $\begin{array}{l}\text { PET responders } \\
(N=69)\end{array}$ & $\begin{array}{l}\text { Group A } \\
(N=48)\end{array}$ & $\begin{array}{l}\text { Group B } \\
(N=25)\end{array}$ & $\begin{array}{l}\text { Total } \\
(N=142)\end{array}$ \\
\hline $\begin{array}{l}\text { Premature end-of-study } \\
\text { Reasons for discontinuation }\end{array}$ & $8(11.6 \%)$ & $10(20.8 \%)$ & $3(12.0 \%)$ & $21(14.8 \%)$ \\
$\mathrm{n}$ & 7 & 10 & 3 & 20 \\
Consent withdrawal & $1(14.3 \%)$ & $5(50.0 \%)$ & 0 & $6(30.0 \%)$ \\
Death & 0 & $2(20.0 \%)$ & $1(33.3 \%)$ & $3(15.0 \%)$ \\
Major protocol deviation & $1(14.3 \%)$ & $1(10.0 \%)$ & 0 & $2(10.0 \%)$ \\
Lost to follow up & $1(14.3 \%)$ & $1(10.0 \%)$ & 0 & $2(10.0 \%)$ \\
Other reason & $4(57.1 \%)^{*}$ & $1(10.0 \%)^{\dagger}$ & $2(66.7 \%)^{\ddagger}$ & $7(35.0 \%)$ \\
Missing data & 1 & 0 & 0 & 1 \\
\hline
\end{tabular}

* Blood glucose not measured at baseline and PET $n^{\circ} 2$ not performed as per Protocol $(n=1)$ discovery of a non-inclusion criterion ( 1.5 months after inclusion; $n=1)$, bone metastasis diagnosed later $(n=1)$, and patient diagnosed M1 after the PET scan and withdrawn at Sponsor's request $(n=1)$;

$\dagger$ Patient moved abroad $(n=1)$;

* Patient included in another clinical trial $(n=2)$.

\section{Results}

\subsection{Initial analysis}

Overall, 176 patients with HER2-positive breast cancer were screened of which 152 patients were included and received two cycles of neoadjuvant therapy (Fig. 1). Ten patients were excluded prior to treatment arm assignment. In total, 142 patients were assigned to a treatment group (ITT population). The baseline and demographic characteristics of the ITT population have been reported previously; [8] patients were well balanced between groups with respect to tumour characteristics that could affect pCR.

Of the 142 patients in the ITT population, 69 (48.6\%) were PET-predicted responders and continued to receive standard docetaxel plus trastuzumab. The remaining 73 (51.4\%) patients were PET-predicted poor-responders and were randomised (2:1) to docetaxel, trastuzumab, and bevacizumab ( $n=48$; Group A) or docetaxel plus trastuzumab ( $n=25$; Group B). One hundred and thirty-nine (97.9\%) patients underwent phase 2 of neoadjuvant treatment, 133 patients underwent surgery, 128 underwent radiotherapy, 132 patients entered the adjuvant period, and 133 patients entered the long-term follow-up phase (123 patients who completed the adjuvant phase and 10 patients who discontinued treatment but entered the follow-up phase).

Initial mean (SD) SUVmax of the affected breast after the first PET scan was lower for predicted poor-responders in Group A and B
$(6 \bullet 68[4 \bullet 21]$ and $7 \bullet 19$ [4•87], respectively) compared with PET responders $(10 \bullet 09[5 \bullet 06])$. Central review determined a pCR rate of 53•6\% (37/69; 95\% CI 41•2-65•7) for PET responders. In poor-responders, the addition of bevacizumab to cycles $4-6$ of neoadjuvant chemotherapy increased the proportion of patients in Group A achieving a pCR to $43 \bullet 8 \%(21 / 48 ; 95 \%$ CI $29 \bullet 5-58 \bullet 8)$ compared with $24 \%$ (6/25; 95\% CI 9•4-45•1) for patients in Group B receiving neoadjuvant docetaxel plus trastuzumab alone [8].

\subsection{Patient disposition during follow-up analysis}

In total, 121/142 patients completed the 5-year follow-up period. The reasons for premature study discontinuation for the 21 patients who did not reach the 5-year follow-up are shown in Table 1.

After a median (range) follow-up of 5.2 years (range 0.1 to 6.5 years), three patients (two in Group A, one in Group B) had died, three patients (one in Group A, two in Group B) experienced local relapse, and seven patients (four in the PET responders group, three in Group B) experience distant relapse.

\subsubsection{Disease-free survival}

At 5-year follow-up, 17 DFS events were reported: four (8.3\%) in Group A, six (24.0\%) in Group B, and seven (10.1\%) in the PET responders. The DFS rates at 5 years were $90.5 \%$ (95\% CI: $80.0-95.6 \%$ ) in PET responders, $90.2 \%$ (95\% CI: 75.9-96.2\%) in Group A and 76.0\%

Table 2

Analysis of time-to-event efficacy endpoints.

\begin{tabular}{|c|c|c|c|}
\hline & $\begin{array}{l}\text { PET responders } \\
(N=69)\end{array}$ & $\begin{array}{l}\text { Group A } \\
(N=48)\end{array}$ & $\begin{array}{l}\text { Group B } \\
(N=25)\end{array}$ \\
\hline \multicolumn{4}{|l|}{ DFS } \\
\hline Number of events, n (\%) & $7(10 \cdot 1)$ & $4(8 \cdot 3)$ & $6(24 \cdot 0)$ \\
\hline Number of censored observations, $\mathrm{n}(\%)$ & $62(89 \cdot 9)$ & $44(91 \cdot 7)$ & $19(76 \cdot 0)$ \\
\hline 5-year DFS rate,\% $(95 \% \mathrm{CI})^{*}$ & $90 \cdot 5(95 \cdot 6,80 \cdot 0)$ & $90 \cdot 2(96 \cdot 2,75 \cdot 9)$ & $76 \cdot 0(88 \cdot 4,54 \cdot 2)$ \\
\hline \multicolumn{4}{|l|}{ LRFI } \\
\hline Number of events & $3(4 \cdot 3)$ & $1(2 \cdot 1)$ & $2(8 \cdot 0)$ \\
\hline Number of censored observations, $\mathrm{n}(\%)$ & $66(95 \cdot 7)$ & $47(97 \cdot 9)$ & $23(92 \cdot 0)$ \\
\hline 5-year LRFI rates,\% $(95 \% \mathrm{CI})^{*}$ & $94 \cdot 8(98 \cdot 3,84 \cdot 6)$ & $97 \cdot 6(99 \cdot 7,83 \cdot 9)$ & $90 \cdot 9(97 \cdot 6,68 \cdot 3)$ \\
\hline \multicolumn{4}{|l|}{ DDFI } \\
\hline Number of events & $4(5 \cdot 8)$ & $0(0 \cdot 0)$ & $3(12 \cdot 0)$ \\
\hline Number of censored observations, $\mathrm{n}(\%)$ & $65(94 \cdot 2)$ & $48(100 \cdot 0)$ & $22(88 \cdot 0)$ \\
\hline 5-year DDFI rates,\% $(95 \% \mathrm{CI})^{*}$ & $95 \cdot 5(98 \cdot 5,86 \cdot 7)$ & $100(100,100)$ & $86 \cdot 9(95 \cdot 6,64 \cdot 5)$ \\
\hline \multicolumn{4}{|l|}{ OS } \\
\hline Number of events & $0(0 \cdot 0)$ & $2(4 \cdot 2)$ & $1(4 \cdot 0)$ \\
\hline Number of censored observations, $\mathrm{n}(\%)$ & $69(100 \cdot 0)$ & $46(95 \cdot 8)$ & $24(96 \cdot 0)$ \\
\hline 5-year OS rates,\% $(95 \% \mathrm{CI})^{*}$ & $100(100,100)$ & $95 \cdot 1(98 \cdot 7,81 \cdot 7)$ & $95 \cdot 8(99 \cdot 4,73 \cdot 9)$ \\
\hline
\end{tabular}

* Derived from Kaplan-Meier estimates. 
A

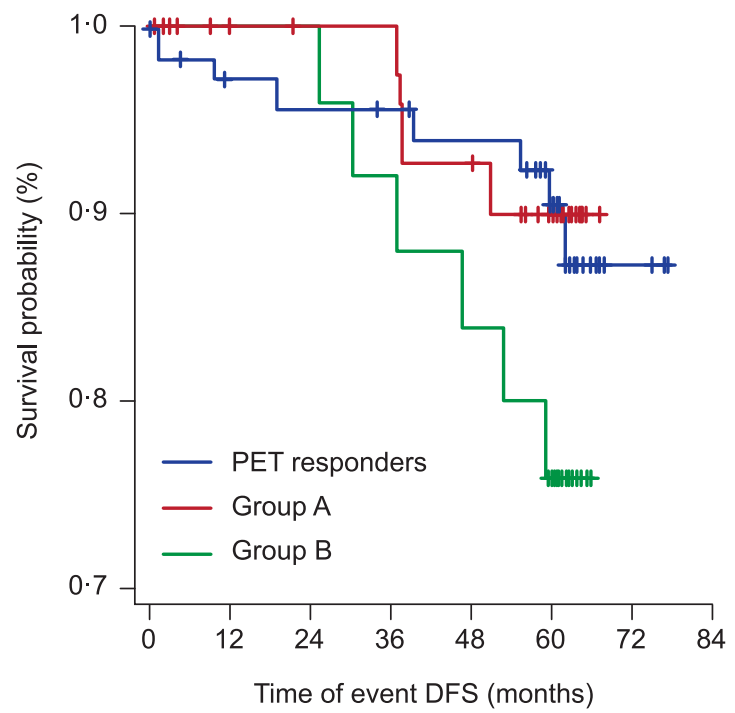

Number at risk (censured)

PET responders $69(0) \quad 63(4) \quad 62(4) \quad 61(5) \quad 59(6) \quad 48(15) \quad 3(59) \quad 0(62)$

Group A $48(0) \quad 42(6) \quad 41(7) \quad 41(7) \quad 38(7) \quad 31(13) \quad 0(44)$

Group B $25(0) \quad 25(0) \quad 25(0) \quad 23(0) \quad 21(0) \quad 17(2) \quad 0(19)$

C

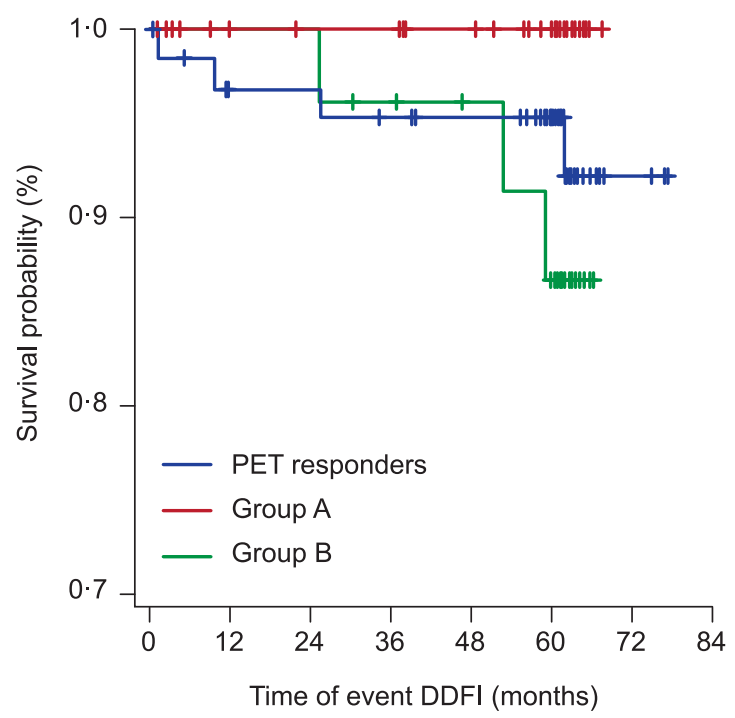

Number at risk (censured)

PET responders $69(0) \quad 63(4) \quad 63(4) \quad 61(5) \quad 59(7) \quad 48(18) \quad 3(62) \quad 0(65)$

Group A $48(0) \quad 42(6) \quad 41(7) \quad 41(7) \quad 38(10) \quad 31(17) \quad 0(48)$

Group B $25(0) \quad 25(0) \quad 25(0) \quad 23(1) \quad 21(3) \quad 17(5) \quad 0(22)$
B

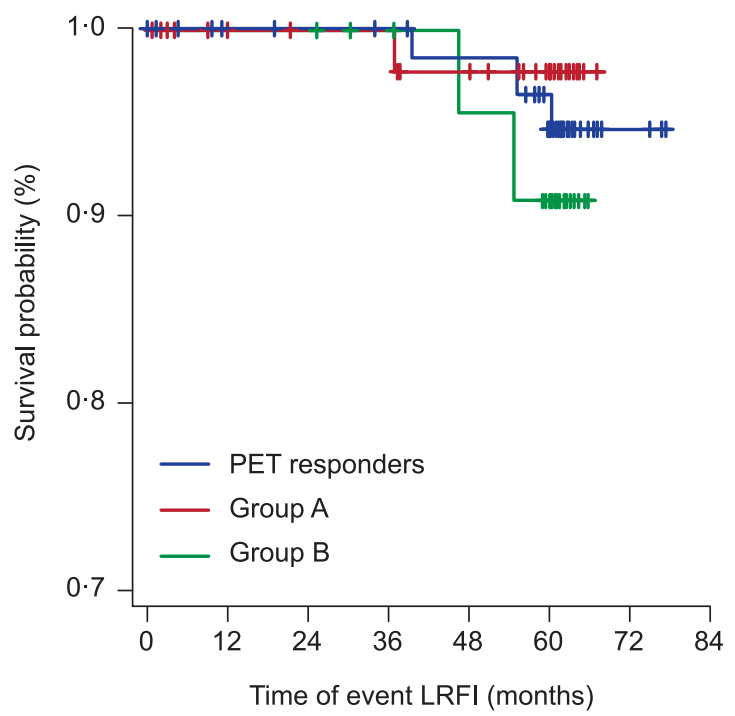

Number at risk (censured)

PET responders $69(0) \quad 63(6) \quad 62(7) \quad 61(8) \quad 59(9) \quad 48(18) \quad 3(63) \quad 0(66)$

Group A $48(0) \quad 42(6) \quad 41(7) \quad 41(7) \quad 38(9) \quad 31(16) \quad 0(47)$

Group B $25(0) \quad 25(0) \quad 25(0) \quad 23(2) \quad 21(3) \quad 17(6) \quad 0(23)$

D

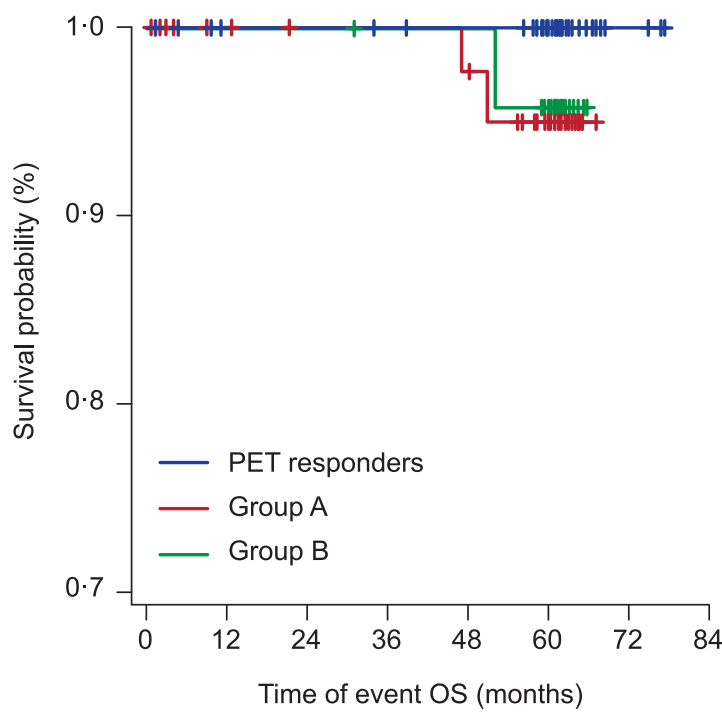

Number at risk (censured)

PET responders $69(0) \quad 63(6) \quad 63(6) \quad 62(7) \quad 61(8) \quad 50(19) \quad 3(66) \quad 0(69$

Group A $48(0) \quad 43(5) \quad 41(7) \quad 41(7) \quad 40(7) \quad 32(14) \quad 0(46)$

Group B $25(0) \quad 25(0) \quad 25(0) \quad 24(1) \quad 24(1) \quad 20(4) \quad 0(24)$

Fig. 3. Kaplan-Meier curves for (A) DFS, (B) LRFI, (C) DDFI, and (D) OS events. Y axes of the figures have been expanded.

(95\% CI: $54.2-88.4 \%$ ) in Group B (Table 2 and Fig. 3A). Median DFS was not reached in all arms.

\subsubsection{Local-relapse-free interval, distant disease-free interval, and} overall survival

Overall, LRFI, DDFI, and OS events were scarce and the median value for these long-term efficacy outcomes not reached in all arms. The 5-year estimates for LRFI, DDFI, and OS are shown in Table 2 and Fig. 3B-D. One (2.1\%) LRFI event was reported in Group A, two (8.0\%) events in Group B, and three (10.1\%) events in the PET responders. Seven DDFI events were reported in total: three (12.0\%) events in
Group B and four (5.8\%) events in the PET responders. For OS, two (4.2\%) events were reported in Group A, one (4.0\%) event in Group B, and no events in the PET responders.

\subsubsection{Post hoc sensitivity analysis of disease-free survival}

There was no apparent difference in DFS between Group A and Group B in the sensitivity analysis of DFS where patients who discontinued the study early (for reasons given in Table 1) were considered to be treatment failures. The 5-year DFS rates were $82.4(95 \% \mathrm{CI}$ : 71.1-89.6) for PET responders, 74.8 (59.9-84.9) for Group A, and 
Table 3

Summary of treatment emergent adverse events (preferred terms) during the adjuvant stage.

\begin{tabular}{|c|c|c|c|}
\hline & $\begin{array}{l}\text { PET responders } \\
(N=65)\end{array}$ & $\begin{array}{l}\text { Group A } \\
(N=43)\end{array}$ & $\begin{array}{l}\text { Group B } \\
(N=24)\end{array}$ \\
\hline Any AE, n (\%) & $64(98 \cdot 4)$ & $43(100)$ & $24(100)$ \\
\hline \multicolumn{4}{|l|}{$\begin{array}{l}\text { AEs occurring in } \geq 20 \% \text { of } \\
\text { patients in at least one group }\end{array}$} \\
\hline Radiation skin injury & $46(70 \cdot 8)$ & $23(53 \cdot 5)$ & $19(79 \cdot 2)$ \\
\hline Arthralgia & $14(21 \cdot 5)$ & $12(27 \cdot 9)$ & $8(33 \cdot 3)$ \\
\hline Lymphocele & $11(16 \cdot 9)$ & $10(23 \cdot 3)$ & $7(29 \cdot 2)$ \\
\hline Hot flash & $16(24 \cdot 6)$ & $7(16 \cdot 3)$ & $8(33 \cdot 3)$ \\
\hline Asthenia & $8(12 \cdot 3)$ & $6(14 \cdot 0)$ & $5(20 \cdot 8)$ \\
\hline Myalgia & $9(13 \cdot 8)$ & $3(7 \cdot 0)$ & $5(20 \cdot 8)$ \\
\hline Any Grade $\geq 3 \mathrm{AE}$ & $11(16 \cdot 9)$ & $11(25 \cdot 6)$ & $3(12 \cdot 5)$ \\
\hline \multicolumn{4}{|l|}{$\begin{array}{c}\text { Grade } \geq 3 \mathrm{AE} \text { occurring in } \geq 4 \% \text { of } \\
\text { patients in at least one group }\end{array}$} \\
\hline Postoperative wound infection & 0 & $3(7 \cdot 0)$ & 0 \\
\hline Lymphocele & $1(1 \cdot 5)$ & $2(4 \cdot 7)$ & 0 \\
\hline Radiation skin injury & $4(6 \cdot 2)$ & $1(2 \cdot 3)$ & $1(4 \cdot 2)$ \\
\hline Post procedural infection & 0 & 0 & $1(4 \cdot 2)$ \\
\hline Cholelithiasis & 0 & 0 & $1(4 \cdot 2)$ \\
\hline Any serious AE & $5(7 \cdot 7)$ & $8(18 \cdot 6)$ & $3(12 \cdot 5)$ \\
\hline Postoperative wound infection & 0 & $3(7 \cdot 0)$ & 0 \\
\hline Impaired healing & 0 & $2(4 \cdot 7)$ & 0 \\
\hline Localised infection & 0 & $1(2 \cdot 3)$ & 0 \\
\hline Lung infection & 0 & $1(2 \cdot 3)$ & 0 \\
\hline Mycoplasma infection & 0 & $1(2 \cdot 3)$ & 0 \\
\hline Arthralgia & 0 & $1(2 \cdot 3)$ & 0 \\
\hline Fibromyalgia & 0 & $1(2 \cdot 3)$ & 0 \\
\hline Lymphocele & 0 & $1(2 \cdot 3)$ & 0 \\
\hline Device related infection & $1(1 \cdot 5)$ & 0 & 0 \\
\hline Post procedural infection & 0 & 0 & $1(4 \cdot 2)$ \\
\hline Breast prosthesis removal & 0 & 0 & $1(4 \cdot 2)$ \\
\hline Arrhythmia & $1(1 \cdot 5)$ & 0 & 0 \\
\hline Palpitations & $1(1 \cdot 5)$ & 0 & 0 \\
\hline Bronchogenic cyst & $1(1 \cdot 5)$ & 0 & 0 \\
\hline Pancreatitis & 0 & 0 & $1(4 \cdot 2)$ \\
\hline Cholelithiasis & 0 & 0 & $1(4 \cdot 2)$ \\
\hline Breast cancer & $1(1 \cdot 5)$ & 0 & 0 \\
\hline Syncope & $1(1 \cdot 5)$ & 0 & 0 \\
\hline
\end{tabular}

Data show the number of patients (percent out of patients in that group) who experienced each of the listed adverse event $(\mathrm{AE})$.

76.0 (54.2-88.4) for Group B (Supplementary Table 1 and Supplementary Figure 1).

\subsection{Adverse events during the adjuvant stage}

A total of 132 patients received at least one dose of trastuzumab during the adjuvant phase. Overall, 913 emergent AEs were reported during the adjuvant phase in $131 / 132$ (99.2\%) patients (Table 3). No patient died due to an $\mathrm{AE}$ or discontinued trastuzumab due to an $\mathrm{AE}$ during the adjuvant phase. The most common AEs were radiation skin injury (91 events in 88 patients, 66.7\%), arthralgia (36 events in 34 patients, $25.8 \%$ ), hot flush (31 events in 31 patients, 23.5\%), and lymphocele (29 events in 28 patients, $21.2 \%$ ). Most events were mild or moderate in intensity (95•8\%); 30 (3.6\%) events were Grade 3 and 5 (0.6\%) were Grade 4. In total, 3.9\% of emergent AEs were judged to be treatment related and $2.5 \%$ of emergent AEs were assessed as serious. The only serious $\mathrm{AE}$ occurring in $>2 \%$ of patients overall was postoperative wound infection. The proportion of patients that experienced a serious AE during the adjuvant period was slightly higher in Group A (18.6\%) compared with Group B (12.5\%) and PET responders (7.7\%). Four serious AEs (two events of impaired healing, one post-operative wound infection, and one localised infection) occurring in three (7.0\%) patients in Group A were considered related to bevacizumab.

Three (2.3\%) patients, all PET responders, experienced four emergent cardiac events during the adjuvant period: arrhythmia in two patients, palpitations in one patient, and supraventricular extrasystoles in one patient. All events were Grade $\leq 2$ and one event of arrhythmia and one event of palpitations were assessed as serious. No patient developed heart failure or had new LVEF decrease during the study.

\section{Discussion}

The AVATAXHER study showed that early PET assessment identified patients with HER2-positive breast cancer who will responded poorly to neoadjuvant docetaxel plus trastuzumab therapy, and that the addition of bevacizumab to neoadjuvant therapy improved $\mathrm{PCR}$ rates in PET-predicted poor responders [8]. The pre-planned 5-year follow-up analysis reported here shows that the improvement in $\mathrm{pCR}$ translated into numerically greater DFS rates; however, the study was not powered to detect a significant difference between treatment arms in this secondary study endpoint, and a difference in DFS between patients treated with or without neoadjuvant bevacizumab was not confirmed in the DFS sensitivity analysis.

Adding bevacizumab to neoadjuvant chemotherapy has been shown to improve pCR rates in patients with HER2-negative breast cancer in the ARTemis, [24] GeparQuinto, [18,25] and NSABP B$40^{17,21}$ studies, as well as in meta-analyses, [26] but in general, these improvements in $\mathrm{PCR}$ have not translated into improved long-term outcomes. The NSABP B-40 trial found that the addition of bevacizumab to neoadjuvant and adjuvant chemotherapy significantly improved PCR in patients with HER2-negative, hormone receptor positive tumours [17] and this improvement in PCR was associated with higher OS but not DFS [21]. Adding bevacizumab anthracyclineand/or taxane-based chemotherapy did not significantly improve invasive DFS or OS in the phase III BEATRICE trial in patients with early triple-negative breast cancer [27]. Interestingly, a phase II study in HER2-positive breast cancer (NSABP FB-5 trial) showed that the addition of bevacizumab to treatment resulted in similar PCR rates to chemotherapy plus trastuzumab alone with high 5-year recurrencefree survival and OS rates [23]. In the present study, the PET-predicted poor-responders who received neoadjuvant bevacizumab had a similar DFS rate at 5-years to PET-predicted responders $(90.2 \%$ and $90.5 \%$, respectively) which was higher than that of the PET-predicted poor-responders who did not receive neoadjuvant bevacizumab (76.0\%). However, these DFS data were not compared statistically in this phase II trial and despite the large numerical difference in DFS rates observed between the PET-predicted poor-responders treated with or without neoadjuvant bevacizumab, there was a wide overlapping of confidence intervals. When patients who discontinued the study early (predominantly due to patients consent withdrawal) were considered as treatment failures, there was no difference in DFS between patients treated with or without neoadjuvant bevacizumab. This does not distract from the main novel finding of the AVATAXHER study which was that PET could be used to identify early those patients who responded poorly to a homogenous anthracycline-free treatment regimen. The DFS rates in PET responders observed in this study compare favourably with those reported in contemporary studies of patients with HER2-positive breast cancer treated with trastuzumab-containing therapy [2,28-30].

We used change of SUVmax as the PET metric in this study to facilitate the comparison of PET data obtained from different study centres in this multicentre study; this parameter has been shown to be robust and correlate well with pCR [31]. However, other PET variables such as SUVpeak have also been used as predictors of neoadjuvant chemotherapy response and prognosis in breast cancer. At 6year follow-up, SUVmax on early PET was shown to independently correlate with OS in patients with breast cancer receiving neoadjuvant chemotherapy [32]. In patients with operable HER2-positive and triple negative breast cancer, the SUVmax determined by PET after neoadjuvant chemotherapy predicted pCR and significantly correlated with recurrence-free survival and recurrence [6]. Volumebased metabolic PET variables have also been shown to be good predictors of both neoadjuvant chemotherapy response and prognosis in 
locally advanced breast cancer [33]. Together, these data suggest that the use of PET in neoadjuvant therapy can predict long-term outcomes for breast cancer patients.

The tolerance profile of study treatments was judged clinically to be acceptable in the adjuvant stage of the AVATAXHER study. The addition of bevacizumab to neoadjuvant chemotherapy resulted in a greater overall number of Grade $\geq 3 \mathrm{AEs}$ observed during the adjuvant period in Group A compared to Group B, with most common Grade $\geq 3$ AE observed in Group A being postoperative wound infection. This increase in Grade 3/4 toxicities is as expected from previously reported studies in HER2-negative breast cancer in which increases in cases Grade 3/4 neutropenia were reported [26,34]. Previous studies have suggested that the addition of bevacizumab to treatment may be associated with increased cardiac events [35]. The AVATAXHER study was an open-label study, which could influence the reporting of AEs; however, the incidence of cardiac events during the adjuvant period was low: all events were Grade $\leq 2$ and occurred in the PET responders. Similarly, long-term follow-up of the HERA trial reported a low incidence of cardiac events, the majority of which were reversible, in HER2-positive breast cancer patients treated with adjuvant trastuzumab [36].

This trial has several other limitations. Events for LRFI, DDFI, and OS were scarce across all three treatment arms. Furthermore, the treatment regimen used in the AVATAXHER study are of limited relevance to clinical practice today. The subsequent approval of pertuzumab and trastuzumab emtansine (T-DM1) changed the paradigm for neoadjuvant treatment of breast cancer, and while anthracyclinefree chemotherapy regimens were being explored at the time of the AVATAXHER study design, the standard combination in neoadjuvant therapy comprises anthracyclines and taxanes. In addition, the acceptability of the tolerability of the treatment regimen was based on clinician judgement whereas the inclusion of patient-reported quality of life measures could have provided insight into the patient's opinion of the tolerability of the regimen. Finally, as this was a Phase II study using a highly selected patient population, the results reported here are not generalisable to other populations.

In conclusion, early PET can identify patients with HER2-positive breast cancer who will responded poorly to neoadjuvant therapy and that the addition of bevacizumab to neoadjuvant docetaxel plus trastuzumab therapy improves the $\mathrm{pCR}$ rate. This improvement in $\mathrm{PCR}$ rate does not translate into long-term improvements in DFS.

\section{Declaration of Competing Interest}

$\mathrm{BC}$ reports personal fees and non-financial support from Roche Laboratories France, personal fees from Bristol Myers Laboratories France, and personal fees from AstraZeneca Laboratories.

JYP declares research funding received by his institution from Roche and Menarini Silicon Biosystems; honoraria received by his institution from Roche and Sanofi; and personal fees from Roche, Novartis, AstraZeneca, Pfizer, Amgen, Ipsen, Lilly, and Genomic Health.

TP is a member of scientific boards for Novartis, Lilly and Pfizer.

TB reports personal fees and non-financial support from Roche, Novartis, AstraZeneca, and Pfizer; and personal fees from Seattle Genetics, outside the submitted work.

DC reports honorarium from Roche, Pfizer, Novartis, Sanofi, and AstraZeneca.

GP acted as consultant for Chugai, Novartis and Shire (Takeda), with remunerations received by his institution. GP's research team received grants for academic studies from Amgen, Genzyme (Sanofi), Lilly, Merck, Novartis, and Roche Pharma.

AF and JD are full-time employees of Roche Pharma France.

$\mathrm{KK}, \mathrm{J}-\mathrm{MF}$, and PG report personal fees from Roche during the conduct of the study.
LA reports personal fees and non-financial support from Roche Laboratories France and Bristol Myers Laboratories France, and personal fees from AstraZeneca Laboratories and Merck.

M-AM-R, FLD, P-FD, M-PC, CB, J-BP, GT, and AB-R declare no conflicts of interest.

\section{Role of the funding source}

The funder of the study (Roche France) supplied trastuzumab and bevacizumab and had a role in the design and conduct of the study; collection, management, and analysis of data (including statistical analyses); interpretation of findings; and writing of the report. The corresponding author had full access to all the data and the final responsibility to submit for publication. All authors were involved in the critical review of the manuscript during its development and approved the final version for submission.

\section{Acknowledgements}

This study was sponsored by Roche France. We thank the patients and their families for their participation in this study, the study management team and staff at individual study sites, Isabelle Josse (Senior Statistician, Aixial, Boulogne-Billancourt, France) for performing the statistical analyses, and Bernard Asselain (Department of Statistics, Institut Curie, Paris, France) for study design discussion. Editorial assistance for the preparation of this article was provided by Jamie Ashman and Megan Christian of Prism Ideas, funded by Roche France.

\section{Author contributions}

BC, J-YP, M-AM-R, J-MF, TP, FLD, P-FD, TB, DC, CB, and J-BP recruited and managed patients in the study and participated in data collection.

BC, J-YP, DC, GP, M-PC, GT, LA, PG, KK, and AB-R participated in protocol development. BC, J-YP, JD, AF (from Roche), and LA analysed and interpreted the data.

$\mathrm{BC}, \mathrm{AF}$ (from Roche), JD, JA, and MC prepared the report.

All authors were given the opportunity to comment on the draft report and saw and approved the final version.

\section{Data sharing statement}

ROCHE, the sponsor of the study, and Dr Coudert, the principal investigator, agree to the principle of data sharing with third parties. Individual participant data that underlie the results reported in this article, after de-identification (text, tables, figures, and appendices) will be available as the study protocol, statistical analysis plan, clinical study report, and analytic code. Roche has already accepted to share the individual data with the Early Breast Cancer Trialists' Collaborative Group (EBCTCG) meta-analysis group.

\section{Supplementary materials}

Supplementary material associated with this article can be found in the online version at doi:10.1016/j.eclinm.2020.100566.

\section{References}

[1] Buzdar AU, Ibrahim NK, Francis D, et al. Significantly higher pathologic complete remission rate after neoadjuvant therapy with trastuzumab, paclitaxel, and epirubicin chemotherapy: results of a randomized trial in human epidermal growth factor receptor 2-positive operable breast cancer. J Clin Oncol 2005;23:3676-85.

[2] Gianni L, Pienkowski T, Im YH, et al. 5-year analysis of neoadjuvant pertuzumab and trastuzumab in patients with locally advanced, inflammatory, or early-stage HER2-positive breast cancer (NeoSphere): a multicentre, open-label, phase 2 randomised trial. Lancet Oncol 2016;17:791-800. 
[3] Humbert O, Riedinger JM, Vrigneaud JM, et al. 18F-FDG PET-derived tumor blood flow changes after 1 cycle of neoadjuvant chemotherapy predicts outcome in triple-negative breast cancer. J Nucl Med 2016;57:1707-12

[4] Garcia Garcia-Esquinas MA, Arrazola GJ, Garcia-Saenz JA, et al. Predictive value of PET-CT for pathological response in stages II and III breast cancer patients following neoadjuvant chemotherapy with docetaxel. Rev Esp Med Nucl Imagen Mol 2014;33:14-21

[5] Gebhart G, Gamez C, Holmes E, et al. 18F-FDG PET/CT for early prediction of response to neoadjuvant lapatinib, trastuzumab, and their combination in HER2positive breast cancer: results from Neo-ALTTO. J Nucl Med 2013;54:1862-8.

[6] Akimoto E, Kadoya T, Kajitani K, et al. Role of (18)F-PET/CT in predicting prognosis of patients with breast cancer after neoadjuvant chemotherapy. Clin Breast Cancer 2018;18:45-52.

[7] Groheux D, Martineau A, Teixeira L, et al. (18)FDG-PET/CT for predicting the outcome in ER+/HER2- breast cancer patients: comparison of clinicopathological parameters and PET image-derived indices including tumor texture analysis. Breast Cancer Res 2017:19:3.

[8] Coudert B, Pierga JY, Mouret-Reynier MA, et al. Use of [(18)F]-FDG PET to predict response to neoadjuvant trastuzumab and docetaxel in patients with HER2-positive breast cancer, and addition of bevacizumab to neoadjuvant trastuzumab and docetaxel in [(18)F]-FDG PET-predicted non-responders (AVATAXHER): an openlabel, randomised phase 2 trial. Lancet Oncol 2014;15:1493-502.

[9] Scheuer W, Friess T, Hasmann M. Enhanced antitumour effect by combination of HER2-targeting antibodies with bevacizumab in a human breast cancer xenograft model. Eur J Cancer Suppl 2006;4:66.

[10] Zhao M, Pan X, Layman R, et al. A Phase II study of bevacizumab in combination with Trastuzumab and docetaxel in HER2 positive metastatic breast cancer. Invest New Drugs 2014;32:1285-94.

[11] Gianni L, Romieu GH, Lichinitser M, et al. AVEREL: a randomized phase III Trial evaluating bevacizumab in combination with docetaxel and trastuzumab as firstline therapy for HER2-positive locally recurrent/metastatic breast cancer. J Clin Oncol 2013;31:1719-25.

[12] Martin M, Makhson A, Gligorov J, et al. Phase II study of bevacizumab in combination with trastuzumab and capecitabine as first-line treatment for HER 2-positive locally recurrent or metastatic breast cancer. Oncologist 2012;17:469-75.

[13] Untch M, Fasching PA, Konecny GE, et al. Pathologic complete response after neoadjuvant chemotherapy plus trastuzumab predicts favorable survival in human epidermal growth factor receptor 2-overexpressing breast cancer: results from the TECHNO trial of the AGO and GBG study groups. J Clin Oncol 2011;29:3351-7.

[14] Cortazar P, Zhang L, Untch M, et al. Pathological complete response and long-term clinical benefit in breast cancer: the CTNeoBC pooled analysis. Lancet 2014;384:164-72.

[15] Ohzawa H, Sakatani T, Niki T, Yasuda Y, Hozumi Y. Pathological responses and survival of patients with human epidermal growth factor receptor 2-positive breast cancer who received neoadjuvant chemotherapy including trastuzumab. Breast Cancer 2014;21:563-70.

[16] Korn EL, Sachs MC, McShane LM. Statistical controversies in clinical research: assessing pathologic complete response as a trial-level surrogate end point for early-stage breast cancer. Ann Oncol 2016;27:10-5.

[17] Bear HD, Tang G, Rastogi P, et al. Bevacizumab added to neoadjuvant chemotherapy for breast cancer. N Engl J Med 2012;366:310-20.

[18] von Minckwitz G, Eidtmann H, Rezai M, et al. Neoadjuvant chemotherapy and bevacizumab for HER2-negative breast cancer. N Engl J Med 2012;366:299-309.

[19] Sikov WM, Berry DA, Perou CM, et al. Impact of the addition of carboplatin and/o bevacizumab to neoadjuvant once-per-week paclitaxel followed by dose-dense doxorubicin and cyclophosphamide on pathologic complete response rates in stage II to III triple-negative breast cancer: CALGB 40603 (Alliance). J Clin Oncol 2015;33:13-21.
[20] Earl HM, Hiller L, Dunn JA, et al. Efficacy of neoadjuvant bevacizumab added to docetaxel followed by fluorouracil, epirubicin, and cyclophosphamide, for women with HER2-negative early breast cancer (ARTemis): an open-label, randomised, phase 3 trial. Lancet Oncol 2015;16:656-66.

[21] Bear HD, Tang G, Rastogi P, et al. Neoadjuvant plus adjuvant bevacizumab in early breast cancer (NSABP B-40 [NRG Oncology]): secondary outcomes of a phase 3, randomised controlled trial. Lancet Oncol 2015;16:1037-48.

[22] Sikov WM, Berry DA, Perou CM, et al. Event-free and overall survival following neoadjuvant weekly paclitaxel and dose-dense $\mathrm{AC}+$ /- carboplatin and/or bevacizumab in triple-negative breast cancer: outcomes from CALGB 40603 (Alliance). Cancer Res 2016;76 Abstract S2-05.

[23] Smith JW, Buyse ME, Rastogi P, et al. Epirubicin with cyclophosphamide followed by docetaxel with trastuzumab and bevacizumab as neoadjuvant therapy for HER2-positive locally advanced breast cancer or as adjuvant therapy for HER2positive pathologic stage III breast cancer: a phase II trial of the NSABP Foundation Research Group, FB-5. Clin Breast Cancer 2017; 17:48-54.

[24] Earl HM, Hiller L, Dunn J, et al. Disease-free and overall survival at 3.5 years for neoadjuvant bevacizumab added to docetaxel followed by fluorouracil, epirubicin and cyclophosphamide, for women with HER2 negative early breast cancer: aRTemis Trial. Ann Oncol 2017:28:1817-24.

[25] von Minckwitz G, Loibl S, Untch M. et al. Survival after neoadjuvant chemotherapy with or without bevacizumab or everolimus for HER2-negative primary breast cancer (GBG 44-GeparQuinto)†. Ann Oncol 2014;25:2363-72.

[26] Cao L, Yao GY, Liu MF, Chen LJ, Hu XL, Ye CS. Neoadjuvant bevacizumab plus chemotherapy versus chemotherapy alone to treat non-metastatic breast cancer: a meta-analysis of randomised controlled trials. PLoS ONE 2015;10:e0145442.

[27] Bell R, Brown J, Parmar M, et al. Final efficacy and updated safety results of the randomized phase III BEATRICE trial evaluating adjuvant bevacizumab-containing therapy in triple-negative early breast cancer. Ann Oncol 2017;28:754-60.

[28] Giacchetti S, Hamy AS, Delaloge S, et al. Long-term outcome of the REMAGUS 02 trial, a multicenter randomised phase II trial in locally advanced breast cancer patients treated with neoadjuvant chemotherapy with or without celecoxib or trastuzumab according to HER2 status. Eur J Cancer 2017;75:323-32.

[29] Guiu S, Liegard M, Favier L, et al. Long-term follow-up of HER2-overexpressing stage II or III breast cancer treated by anthracycline-free neoadjuvant chemotherapy. Ann Oncol 2011;22:321-8.

[30] von Minckwitz G, Rezai M, Fasching PA. et al. Survival after adding capecitabine and trastuzumab to neoadjuvant anthracycline-taxane-based chemotherapy for primary breast cancer (GBG 40-GeparQuattro). Ann Oncol 2014;25:81-9.

[31] Groheux D, Majdoub M, Sanna A, et al. Early Metabolic Response to Neoadjuvant Treatment: FDG PET/CT Criteria according to Breast Cancer Subtype. Radiology 2015;277:358-71.

[32] Chen S, Ibrahim NK, Yan Y, Wong ST, Wang H, Wong FC. Complete metabolic response on interim (18)F-fluorodeoxyglucose positron emission tomography/ computed tomography to predict long-term survival in patients with breast cancer undergoing neoadjuvant chemotherapy. Oncologist 2017;22:526-34.

[33] Garcia-Vicente AM, Perez-Beteta J, mo-Salas M, et al. Predictive and prognostic potential of volume-based metabolic variables obtained by a baseline (18)F-FDG PET/CT in breast cancer with neoadjuvant chemotherapy indication. Rev Esp Med Nucl Imagen Mol 2018;37:73-9.

[34] Han R, Wang G, Zhang Y, Zhao X. [Efficacy of neoadjuvant chemotherapy combined with bevacizumab versus neoadjuvant chemotherapy alone for Her2-negative breast cancer: a meta-analysis of randomized controlled clinical trials]. Zhejiang Da Xue Xue Bao Yi Xue Ban 2016;45:379-86.

[35] Choueiri TK, Mayer EL, Je Y, et al. Congestive heart failure risk in patients with breast cancer treated with bevacizumab. J Clin Oncol 2011;29:632-8.

[36] de Azambuja E, Procter MJ, van Veldhuisen DJ. et al. Trastuzumab-associated cardiac events at 8 years of median follow-up in the Herceptin Adjuvant trial (BIG 101). J Clin Oncol 2014;32:2159-65. 